\title{
The Study of the Authentic Test Method in the Graduate Design of the Major of Electronic Specialty
}

\author{
Huang Ling, Ma Zhaomin, and Hu Bo \\ College of Electric and Information Engineering, Guangxi University of Science and Technology, Liuzhou, Guangxi, China
} (huangling79hl@163.com)

\begin{abstract}
Authentic assessment is an effective way for promoting the teaching quality in graduate design. The test method is the foundation of authentic assessment. The steps of graduate design of the major of electronic specialty are first introduced. Then the authentic test method of every step in the graduate is discussed. And several noticeable problem of the authentic test method selection in the graduate design of the major of electronic specialty are proposed. It should be helpful for promoting the student's abilities in the graduate design of the major of electronic specialty.
\end{abstract}

Keywords-Authentic test method, graduate design, electronic specialty

\section{电气类专业毕业设计真实性评价方式的研究}

\author{
黄玲 马兆敏 胡波 \\ 广西科技大学电气与信息工程学院，柳州，广西，中国
}

摘 要 真实性评价是提高毕业设计教学质量的有效途径之一, 而评价方式是真实性评价的基础。首先介绍了电气类专业毕业设 计中的步骤, 然后讨论了每个步骤的真实性评价方式, 最后提出了在电气类专业毕业设计真实性评价方式选择中值得注意的几个问题。 这将有助于在电气类专业毕业设计中提高学生的能力。

关键词 真实性评价方式, 毕业设计, 电气类专业

\section{1. 引言}

毕业设计一直是电气类专业学生专业能力培养的重要 教学环节, 所以这一直是电气类专业教学改革的热点 ${ }^{[1][2]}$ 。 在现实教学实践中毕业设计的质量一直无法令人完全满 意, 特别是高等教育进入大众化阶段后尤为明显。而另一 方面, 在进入企业后, 作为预备工程师许多学生通过一段 时间的训练可以达到电气工程师的要求。因此, 按照预备 工程师真实的训练环境对毕业设计进行教学改革成为提高 教学质量的有效途径。

在已有教育理论中, 真实性评价作为一种强调与真实 环境相统一的教学理念被广泛接受, 在电气类的实践教学 中引入真实性评价可有效提高教学质量 ${ }^{[3]}$ 。而在真实性评 价中, 评价方式是基础, 因此需要根据真实性评价理论对 电气类专业毕业设计的评价方式进行教学改革。

广西科技大学 2014 年度教育教学改革立项项目支持

\section{2. 电气类专业毕业设计的步骤}

电气类专业在我国本科专业的定义中一般包括自动 化、电气工程及其自动化、电子科学与技术等专业。这些 专业学生的毕业设计根据完成任务的差异大体分为进行硬 件设计、软件设计、理论研究等几个类型。虽然不同类型 毕业设计题目在毕业设计各个环节的具体内容存在一定的 差异, 但是总体的步骤是相对统一的。一般认为电气类专 业的毕业设计包括文献检索、开题、实验与软硬件开发、 数据处理与测试、论文撰写和答辩 6 个步骤。

文献检索主要是根据课题对已有研究成果进行学习。 这包括已发表论文、专利、设备技术文档等多种资料。根 据毕业设计题目类型的差异侧重点有所不同。

开题主要是明确毕业设计的研究目的、研究内容和研 究方法。开题是在文献检索的基础上对于开展毕业设计的 一个总体设想, 不少情况下开题前还需要进行一些预备性 实验或软硬件设计, 通过总结这些预备性工作结果完成开 
题报告。

实验和软硬件设计就是按开题报告设想的方案进行毕 业设计。这一阶段既是对毕业设计课题的工作, 也是对已 有知识的应用, 因此这一阶段是毕业设计主要的设计工作 阶段。

在完成实验和软硬件设计后, 需要对已完成毕业设计 工作进行评价和测试, 这就是数据处理与测试的阶段。这 一阶段是对前一阶段工作的验证与检查, 如果结果不理想 需要重复、补充、修正实验和软硬件设计阶段的工作。由 于学生初次进行毕业设计如此长时间的专业工作, 这一阶 段往往与前一阶段不断反复, 直至学生的毕业设计工作达 到预期要求。

在完成了基本的毕业设计工作后, 主要就是论文的撰 写。需要按照科技文献的标准, 将所完成的工作撰写成一 篇体系较为完整的毕业设计论文。

在完成论文后, 最后一个步骤就是进行毕业设计答辩。 通过答辩陈述描述自己的工作, 通过回答提问展现自己对 自己所完成工作的理解, 从而体现学生自己在毕业设计过 程所获得专业能力的锻炼。

\section{3. 毕业设计各步骤的真实性评价方式}

毕业设计这一教学环节的设置本意就是模拟工程实践 的真实环境对学生进行训练, 因此在各个步骤的教学内容 上都是与真实工程实践环境相对应的。这就意味这毕业设 计的内容上是基本符合真实性评价的要求的, 但是值得注 意的由于毕业设计需要给出一定的成绩, 所以在评价方式 上往往以学生完成工作的结果为核心, 从而与真实环境相 违背。结合真实环境中预备工程师的训练, 电气类专业毕 业设计各个步骤可采取不同的真实性评价方式。

\section{1 文献检索}

文献检索阶段学生所完成的工作是很难单独评价其效 果的。现有的毕业设计评价方式中往往以学生查阅文献的 能力作为评价内容, 通过对文献检索和阅读的情况进行定 性评价, 所以这样的评价方式与真实环境是不合适的。根 据真实性评价理论, 这一阶段可考虑采取学习档案的评价 方式, 只记录学生在毕业设计阶段应用查阅到文献的情况, 不对单独对学生文献检索结果进行好坏的评价, 而将对文 献查阅好坏的情况与后面实际工作联系起来。

\section{2 开题}

开题是一个学生展现自我学习成果的过程, 根据工程 师培养的真实情况, 这一阶段不宜打击学生的主动性。因 此, 在评价过程中不应强调开题通过与不通过, 而更注重
开题报告中研究目的、研究内容和研究方法等细节, 通过 开题报告会上教师提问的形式给学生形成一个自我反思。 因此该阶段的评价方式可以考虑以学生自我评价为主, 甚 至可以在毕业设计不同的步骤中让学生多次评价自己的开 题报告。

\section{3 实验和软硬件设计与数据处理与测试}

实验和软硬件设计、数据处理与测试这两个阶段是完 成毕业设计的核心阶段。这一阶段是考察学生是否完成设 计的关键, 因此这一阶段应该进行明确的测试。根据学生 的毕业设计题目, 明确测试点及测试标准。这也是工程实 践中项目结题验收不可缺少的环节。

\section{4 论文撰写与答辩}

论文撰写与答辩某种意义上讲既是一个学生学习的过 程, 也是学生展现能力的过程。现有毕业设计中往往以毕 业设计结果作为评价的核心, 评价方式上重结果而轻能力。 而在真实环境中, 用人单位往往更重视在整个设计过程中 学生所体现出来的专业能力, 根据这一能力判断其是否具 备成为电气工程师的潜质。因此, 在这两个阶段的真实性 评价方式中应该注重能力的评价。

\section{4. 评价方式选择中值得注意的问题}

根据真实性评价理论, 结合教学实践, 对于电气类专 业毕业设计各步骤可以得出一定的评价方式, 但是在实际 操作中对于具体的组织形式、评价方式细节却存在巨大差 异, 因此在电气类专业毕业设计评价方式的具体选择中以 下几个问题值得注意。

\section{1 不同类型毕业设计题目之间的差异}

电气类专业毕业设计根据侧重点不同可以分为不同的 类型, 在同样的毕业设计步骤中, 不同类型的题目对学生 的具体工作具有不同的要求。因此, 在选择具体的评价方 式时, 需要根据不同类型的题目设定细节。如在数据处理 和测试阶段, 如何设定测试点, 有时候甚至需要针对每个 毕业设计设置不同的测试方案。

\section{2 评价的目的}

毕业设计的目的是培养学生专业能力, 在真实性评价 中评价的目的是促进学生能力的提高, 因此在每个阶段选 择评价方式时最后都要考虑是否有利于学生能力的提高, 而不能仅考虑评价本身。 


\section{5. 小结}

针对电气类专业毕业设计真实性评价, 讨论了各个步 骤所采取的真实性评价方式及值得注意的问题, 这将为在 电气类专业毕业设计中开展真实性评价提供理论基础。

\section{参考文献(References)}

[1] Hua Honhyan, Chu Suiying. Exploration about the reform of electronic engineering $\&$ automation specialty practice teaching. Journal of Zhengzhou institute of aeronautical industry management (Social Science Edition). vol.24 (1) pp.97-98, 2005

[2] Feng Zengxi, Ren Qingchang, Yu Junqi and He Bo. The practice and study on the graduate design of the major of electrical and intelligent building. Journal of Jilin institute of architecture \& civil engineering. vol.29 (6) pp.61-63 2012

[3] Zhou Yanming, Ma Zhaomin and Qi Baoqi. The study of the authentic assessment in microcontroller unit course design. 2nd International Conference on Education and Sports Education vol.5 74-76 